\title{
A Relationship between nondeterministic Turing machines and 1-inkdot Turing machines with small space
}

\section{$\operatorname{AUTHOR}(\mathrm{S})$ :}

Inoue, Katsushi; Ito, Akira; Takanami, Itsuo

\section{CITATION:}

Inoue, Katsushi ...[et al]. A Relationship between nondeterministic Turing machines and 1-inkdot Turing machines with small space. 数理解析研究所講究録 1992, 790: 1-7

ISSUE DATE:

1992-06

URL:

http://hdl.handle.net/2433/82676

RIGHT: 
A Relationship between nondeterministic Turing machines and 1-inkdot Turing machines with small space

by

Katsushi Inoue, Akira Ito and Itsuo Takanami

井上克司伊藤暁高浪五男

Department of Computer Science and Systems Engineering
Faculty of Engineering
Yamaguchi University
Ube, 755 Japan

Keywords: Computational complexity, space bounded computations, nondeterministic Turing machines, 1-inkdot Turing machines

\section{Introduction and Preliminaries}

We consider the Turing machine model with a two-way, read-only input tape and a separate two-way, read-write worktape [5]. Recently, Ranjan, Chang and Hartmanis [6] introduced a slightly modified Turing machine model, called a 1-inkdot Turing machine. The 1-inkdot Turing machine is a Turing machine with the additional power of marking 1 tape-cell on the input (with an inkdot). This tape-cell is marked once and for all (no erasing) and no more than one dot of ink is available. The ac- 
tion of the machine depends on the current state, the currently scanned input and worktape symbols and the presence of the inkdot on the currently scanned tape-cell. The action consists of moving the heads and making appropriate changes on worktape cells (using the finite control). In addition, the inkdot may be used to mark the currently scanned cell on the input tape if it has not been used already.

A nondeterministic Turing machine $M$ is called strongly (weakly) $L(n)$ space-bounded if for any $n \geq 1$ and for any input w of length $n$, it uses no more than $L(n)$ worktape cells on $w$ (if for any $n \geq 1$ and for any input $w$ of length $n$ accepted by $M$, there is an accepting computation of $M$ on $w$ using no more than $L(n)$ worktape cells). A strongly (weakly) $L(n)$ space-bounded nondeterministic 1 -inkdot Turing machine is defined similarly.

Let strong-NSPACE[L(n)] (strong-NSPACE*[L(n)]) denote the class of languages accepted by strongly $L(n)$ space-bounded nondeterministic Turing machines (1-inkdot Turing machines), and let weak-NSPACE$[L(n)]$ (weak-NSPACE* $[L(n)]$ ) denote the class of languages accepted by weakly $L(n)$ space-bounded nondeterministic Turing machines (1-inkdot Turing machines).

Ranjan, Chang and Hartmanis [6] left the following open problem:

$$
\text { strong-NSPACE[ } \log \log n]=\text { strong-NSPACE*[loglog } n] \text { ? }
$$

This problem was solved by Geffert [4], who proved that the language $\left\{a^{m} b^{n} \mid m \neq n\right\}$ is not in strong-NSPACE[o(log $\left.\left.n\right)\right]$; Ranjan et.al.[6] showed that $\left\{a^{m} b^{n} \mid m \neq n\right\} \in \operatorname{strong-NSPACE*}[\log \log n]$.

"The proof of Geffert" is based on space constructibility, and is valid only for the strong mode of space complexity, because 
the language $\left\{a^{m} b^{n} \mid m \neq n\right\} \in$ weak-NSPACE[ $\left.\log \log n\right][1,3]$.

The present paper gives a new, simple proof for solving the above problem, which is independent of any space constructibility properties, and which can also be applied to the weak mode of space complexity. In fact, we prove the following theorem:

Theorem 1. There is a language $\mathrm{T}$ such that $T \in$ strong-NSPACE* $[\log \log n]$ - weak-NSPACE$[L(n)]$, for any $L(n)=0(\log n)$.

Throughout this paper, we assume that logarithms are base 2 .

\section{2. $\underline{\text { Proof }}$}

Let $T=\left\{B(1) \# B(2) \# \ldots \# B(n) c w 1 c w_{2} c \ldots c w_{k} c c w \in\{0,1, c, \#\}+\mid n \geq 2 \&\right.$ $k \geq 1 \&\left(w, w_{1}, \ldots, w_{k} \in\{0,1\}^{+}\right) \&|w|(i . e .$, the length of $w)=r$ $\left.\log n^{7} \& w \in\left\{w_{1}, \ldots, w_{k}\right\}\right\}$, where for each positive integer $i \geq 1$, $B(i)$ denotes the string in $\{0,1\}^{+}$that represents the integer $i$ in binary notation (with no leading zeros).

We first show that $T$ is in strong-NSPACE* $[\log \log n]$. We consider a strongly loglog $n$ space-bounded nondeterministic 1 inkdot Turing machine $M$ which acts as follows. Suppose that an input string

$\notin \mathrm{y}_{1} \# \mathrm{y}_{2} \# \ldots \ldots \mathrm{y}_{\mathrm{n}} \mathrm{cw} 1 \mathrm{cw} 2 \mathrm{c} \ldots \mathrm{cwk} \mathrm{ccw} \$$

(where $n \geq 2, k \geq 1$, and $y_{i}^{\prime} s, w_{j}^{\prime} s, w$ are all in $\{0,1\}^{+}$, and $\varnothing$ 
(\$) is the left (right) endmarker) is presented to M. (Input strings in the form different form the above can be easily rejected by M.l By using the well-known technique (see Problem 10.2 in [5]), $M$ first marks off $\log \log n$ worktape cells when $\mathrm{y}_{i}=\mathrm{B}(\mathrm{i})$ for each $1 \leq i \leq n$. (Of course, $M$ enters a rejecting state if $y_{i} \neq B(i)$ for some $1 \leq i \leq n$.) $M$ then checks by using loglog $n$ worktape cells that $|w|=r \log \mathrm{n}^{7}$. After that, $M$ nondeterministically chooses some $j(1 \leq j \leq k)$, and marks the symbol c just before $w_{j}$ by the inkdot. M finally checks by using loglog $n$ worktape cells as a counter that $w=w j . M$ accepts the input string only if these checks are all successful. It will be obvious that $M$ accepts the language $T$.

We next show that $T$ is not in weak-NSPACE[o(log $n)]$. Suppose, to the contrary, that $T \in$ weak-NSPACE$[L(n)]$, where $L(n)=o(\log$ n), and let $M$ be a weakly $L(n)$ space-bounded nondeterministic Turing machine accepting $T$. For each $n \geq 1$, let

$$
V(n)=\{B(1) \# B(2) \# \ldots \# B(n) y c c w \mid y \in W(n) \& w \in\{0,1\} r \log n 7\},
$$

where $W(n)=\left\{c w_{1} c w_{2} c \ldots c w_{n} \mid w_{1}, w_{2}, \ldots, w_{n} \in\{0,1\}\ulcorner 10 g n 7\}\right.$. We consider the computations of $M$ on the strings in $V(n)$. Let $r(n)$ be the length of each element in $V(n)$. Then $r(n)=O(n \log n)$. A storage state of $M$ is a combination of the state of the finite control, the non-blank contents of the worktape, and the worktape head position. Let $C(n)$ denote the set of all possible $L(r(n))$ space-bounded storage states of $M$, and let $u(n)$ be the number of elements of $C(n)$. Then $u(n)=s L(r(n)) t^{u(r(n))}$, where $s$ and $t$ are the numbers of states (of the finite control) and worktape symbols of $M$, respectively.

Let $y, y$, be any two strings in $w(n)$. We say that $y$ and $y$, are 
M-equivalent if for each pair of storage states $q, q^{\prime} \in C(n)$, there exists an $L(r(n))$ space-bounded computation in which $M$ enters $\phi B(1) \# \ldots \# B(n) y$ in $q$ (from the right) and exits in $q$ ' (to the right) afterwards if and only if there exists an $L(r(n))$ space-bounded computation in which M enters $\phi$ $B(1) \# \ldots \# B(n) y^{\prime}$ in $q$ and exits in $q^{\prime}$. Clearly there are at most $E(n)=2^{u(n) \times u(n)}$ M-equivalence classes. On the other hand there are at least $2^{n}-1$ different sets of strings from $\{0,1\}\lceil 10 g$ n 7 occuring in elements of $W(n)$. From the assumption that $L(n)=o(\log n)$ and from the fact that $r(n)=O(n \log n)$, we have $2^{n}-1>E(n)$ for $n$ large enough and there are two M-equivalent elements $y$ and $y^{\prime}$ in $W(n)$ such that there is a string $w \in\{0,1\}$ r log $n 7$ which occurs in $y$ but not in $y$ '. Applying now "cutand-paste" technique, one can obtain an input which is not in the language $T$, but is accepted by $M$, a contradiction. This completes the proof of "T weak-NSPACE[o( $\log n)] "$.

\section{Discussions}

The demon machines [6] are ordinary $L(n)$ space-bounded Turing machines having marked of $L(n)$ space on the worktape automatically, at the very beginning of the computation, so that they do not have to worry about constructing the space needed for recognition.

The proof in the previous section actually shows that there is a language $T$ which can be accepted by a strongly loglog $n$ 
space-bounded nondeterministic 1 -inkdot Turing machine but by no weakly o $(\log n)$ space-bounded nondeterministic demon machine. Thus it shows that

$\exists \mathrm{T} \in$ strong-NSPACE* $[\log \log n]$ - weak-NDEMONSPACE$[L(n)]$, for any $L(n)=o(\log n)$.

It will be interesting to investigate a relationship between ASPACE$[L(n)]$ and $\operatorname{ASPACE}^{*}[L(n)]$ for any $L(n)$ such that $L(n) \geq \log \log n$ and $L(n)=o(\log n)$, where $\operatorname{ASPACE}[L(n)]$ (ASPACE* $[L(n)]$ ) denotes the class of languages accepted by $L(n)$ space-bounded alternating Turing machines [2] (L(n) spacebounded alternating 1 -inkdot Turing machines).

Acknowledgment

The authors would like to thank anonymous referees for greatly improving the presentation of the paper.

\section{REFERENCES}

[1] H.Alt and K.Melhorn, Lower bounds for the space complexity of context-free recognition, Proc.3rd Collon Automata, Languages and Programming (Springer, Berlin, 1976)339-354.

[2] A.K.Chandra, D.C.Kozen and L.J.Stockmeyer, Alternation, J.Assoc.Comput.Mach.28(1)(1981)114-133.

[3] R.Freivalds, on time complexity of deterministic and nondeterministic Turing machines, Latvian Math.23 (1979) 158-165. [4] V.Geffert, Nondeterministic computations in sublogarithmic 
space and space constructibility, SIAM Journal on Computing, $20(3)$ (June 1991)484-498.

[5] J.E.Hopcroft and J.D.Ullman, Formal Languages and Their Relation to Automata (Addison-Wesley, Reading,MA, 1969).

[6] D.Ranjan, R. Chang and J.Hartmanis, space bounded computations: review and new separation results, Theoret. Comput.Sci.80(1991)289-302. 